\title{
Automatisierte Schneeflächenbestimmung mit digitalen Multispektraldaten des ERTS-1
}

Mit Hilfe der wiederholten Satellitenaufnahme ist es erstmals möglich, die Veränderungen der Schneedecke über größere Gebiete hinweg festzustellen und zu kartieren. Eine erste Aufgabenstellung umfaßt deshalb die automatisierte Kartierung und Messung der mit Schnee bedeckten Flächen im Gebirge. Es sollen Charakteristika, die diese Flächen im Satellitenbild meßtechnisch kennzeichnen, bestimmt werden. Ziel dieser Arbeit ist die Entwicklung einer Methode, um die Digitaldaten der Multispektralaufnahmen des Erderkundungssatelliten ERTS-1 für die automatische Schneeflächenbestimmung einsetzen zu können. Am
Beispiel eines $20 \times 22 \mathrm{~km}^{2}$ großen Alpen-Ausschnittes (Bergell) der ERTS-Aufnahmeserie 1076-09442 (Fig. 1) vom 7. Oktober 1972 wurde eine konkrete Lösung erarbeitet (Fig. 2).

Da die im Testgebiet berücksichtigten Oberflächenobjekte in verschiedenen Spektralbereichen unterschiedliches Reflexionsverhalten aufweisen, wurde eine Methode angewandt, bei der für die Schneecharakterisierung jeweils simultan die zugehörigen Reflexionswerte aus allen vier MSS-Spektralbereichen beigezogen werden.

Als Verfahren wurde die aus der Statistik bekannte

Tabelle 1: Neue Stichprobensammlung (17 Stichproben)

\begin{tabular}{|c|c|c|c|c|c|c|c|c|}
\hline \multicolumn{2}{|r|}{$\overline{\mathrm{x}}$} & $\sigma$ & & $\overline{\mathrm{x}}$ & $\sigma$ & & $\overline{\mathrm{x}}$ & $\sigma$ \\
\hline A & $\begin{array}{lr}\text { a) } & 118.94 \\
\text { b) } & 118.69 \\
\text { c) } & 108.27 \\
\text { d) } & 41.12\end{array}$ & $\begin{array}{r}14.09 \\
14.20 \\
17.77 \\
9.60\end{array}$ & B & $\begin{array}{r}21.20 \\
11.33 \\
7.08 \\
1.04\end{array}$ & $\begin{array}{l}1.69 \\
1.29 \\
1.11 \\
0.53\end{array}$ & $\mathrm{C}$ & $\begin{array}{l}31.87 \\
31.53 \\
42.02 \\
21.93\end{array}$ & $\begin{array}{l}2.12 \\
2.28 \\
1.82 \\
1.26\end{array}$ \\
\hline D & $\begin{array}{r}13.37 \\
6.80 \\
5.25 \\
1.44\end{array}$ & $\begin{array}{l}1.07 \\
1.24 \\
2.11 \\
1.35\end{array}$ & $\mathrm{~N}$ & $\begin{array}{r}13.56 \\
5.36 \\
2.77 \\
0.01\end{array}$ & $\begin{array}{l}0.66 \\
0.93 \\
0.66 \\
0.11\end{array}$ & $\mathrm{O}$ & $\begin{array}{r}35.62 \\
20.37 \\
7.43 \\
0.29\end{array}$ & $\begin{array}{l}1.13 \\
0.82 \\
0.93 \\
0.45\end{array}$ \\
\hline$P$ & $\begin{array}{l}36.03 \\
35.30 \\
40.78 \\
20.14\end{array}$ & $\begin{array}{l}4.39 \\
4.11 \\
2.62 \\
1.87\end{array}$ & $\mathrm{Q}$ & $\begin{array}{l}24.05 \\
21.56 \\
36.13 \\
20.05\end{array}$ & $\begin{array}{l}2.87 \\
2.96 \\
4.50 \\
2.74\end{array}$ & $\mathrm{R}$ & $\begin{array}{l}19.79 \\
14.16 \\
39.56 \\
23.34\end{array}$ & $\begin{array}{l}1.22 \\
1.46 \\
3.45 \\
2.46\end{array}$ \\
\hline S & $\begin{array}{l}15.85 \\
10.20 \\
19.11 \\
10.22\end{array}$ & $\begin{array}{l}1.05 \\
1.50 \\
2.91 \\
1.92\end{array}$ & $\mathrm{~T}$ & $\begin{array}{r}12.63 \\
5.25 \\
3.77 \\
0.70\end{array}$ & $\begin{array}{l}1.06 \\
1.27 \\
1.04 \\
0.70\end{array}$ & $\mathrm{U}$ & $\begin{array}{r}125.00 \\
125.15 \\
117.75 \\
45.84\end{array}$ & $\begin{array}{r}6.57 \\
6.55 \\
10.82 \\
8.53\end{array}$ \\
\hline V & $\begin{array}{r}23.18 \\
12.84 \\
8.58 \\
1.58\end{array}$ & $\begin{array}{l}2.69 \\
1.64 \\
1.47 \\
0.66\end{array}$ & W & $\begin{array}{r}20.50 \\
11.34 \\
8.16 \\
1.82\end{array}$ & $\begin{array}{l}2.92 \\
2.19 \\
2.86 \\
1.26\end{array}$ & $X$ & $\begin{array}{r}125.58 \\
125.86 \\
116.20 \\
42.28\end{array}$ & $\begin{array}{l}1.01 \\
1.03 \\
4.92 \\
2.37\end{array}$ \\
\hline$Y$ & $\begin{array}{r}29.82 \\
19.66 \\
13.44 \\
3.20\end{array}$ & $\begin{array}{l}1.73 \\
1.90 \\
1.57 \\
0.65\end{array}$ & $\mathrm{Z}$ & $\begin{array}{r}125.41 \\
124.29 \\
104.47 \\
35.59\end{array}$ & $\begin{array}{l}3.42 \\
4.96 \\
7.56 \\
2.53\end{array}$ & & $\begin{array}{l}=\mathrm{Ka} \\
=\mathrm{Ka} \\
=\mathrm{Ka} \\
=\mathrm{Ka}\end{array}$ & $\begin{array}{l}\text { al } 4 \\
\text { al } 5 \\
\text { al } 6 \\
\text { al } 7\end{array}$ \\
\hline
\end{tabular}

Legende:

$\mathrm{SO}=$ Sonnenseitenlage

$\mathrm{SA}=$ Schattenseitenlage

Anzahl Bildpunkte

$A=$ Schnee SO 111

$\mathrm{B}$ - Schnee SA 90

$\mathrm{C}=$ Gras SO 96

$\mathrm{D}=$ Gras SA $\quad 128$

$\mathrm{N}=$ Silsersee SO 75

$\mathrm{O}=$ Gletschersee Albigna SO 72

$\mathrm{P}=$ Gras/Boden SO 84

$Q=$ Gras SO 72

$\mathrm{R}=$ Laubwald SO 99

$\mathrm{S}=$ Nadelwald SO 68

$\mathrm{T}=$ Nadelwald SA $\quad 99$

$U=$ Schnee SO 84

$V=$ Schnee SA 82

$\mathrm{W}=$ Schnee und Fels SA 75

$X$ - Schnee und Eis SO 60

$Y$ - Schnee und Eis SA 75

$\mathrm{Z}=$ Gletscher SO Bildpunkte Total $\overline{1442}$

Dr. Rudolf Gfeller, Geographisches Institut der Universität Zürich, Blümlisalpstraße 10, 8006 Zürich. Dr. K. Seidel, Fotographisches Institut der ETH Zürich, Sonneggstraße 5, 8006 Zürich 
lineare Diskriminanzanalyse benutzt. Die vom Multispektralscanner gelieferten Videodaten standen uns in digitaler Form zur Verfügung. Zuerst wurden aus der Datenmatrix eine Anzahl Objekte als Stichproben abgegrenzt (Tab. 1). Die Trennbarkeit dieser Stichproben beruht mathematisch auf der Tatsache, daß sie im 4-dimensionalen Variablenraum unterschiedliche $\mathrm{Ge}$ biete einnehmen. Zur Berechnung der Trennfunktion dieser Gebiete wurde das am RZETH vorhandene Programm BMD07M verwendet, das die lineare Diskriminanzanalyse schrittweise durchführt.

Es konnte gezeigt werden (Tab. 2), daß sich Schnee in verschiedenen Expositionen zur Sonne allein aufgrund seiner Reflexionscharakteristika in den vier ERTS-MSS-Kanälen bestimmen läßt. Allerdings muß man hierfür eine genügende Anzahl repräsentanter Stichproben auswählen. Mit der errechneten Trennformel läßt sich die Schneedecke aus den Satellitenbildern kartieren, indem man die vom Computer kommenden Klassifikationsresultate wieder bildmäßig dar- stellt. In jedem Fall ist die Qualität der Methode abhängig von der Auswahl der Stichproben, d.h. von deren Abgrenzung nach Lage und Umfang.

In unserem Falle wurden zur Schneeklassierung und -kartierung zwei Stichprobensätze zusammengestellt, wobei der erste für den sogenannten Normalfall dient (trockener Neuschnee, Tab. 3), während der zweite für Bilder verwendet werden kann, deren Aufnahme im Zeitpunkt einer Schneeausaperungsperiode (z.T. feuchter, schmelzender Schnee) erfolgte.

Wichtig ist bei beiden Varianten, daß für die Schneegrenzzone eine Stichprobe genau abgegrenzt werden kann. Die Festlegung der Stichproben sollte darum zum Zeitpunkt der Aufnahme im Felde vorgenommen werden.

Anhand der aus der Diskriminanzanalyse resultierenden Trennfunktion lassen sich alle Bildpunkte einer ERTS-MSS-Bildmatrix in «Schnee» oder «schneefreien Bildhintergrund» einteilen (Fig. 2). Die Klassifikationsmatrix repräsentiert die Summenhäufigkeit der Bild-

Tabelle 2: Klassifikationsmatrix für die in Tabelle 1 aufgeführten Stichproben

\begin{tabular}{|c|c|c|c|c|c|c|c|c|c|c|c|c|c|c|c|c|c|c|}
\hline \multirow{6}{*}{ SSO } & \multirow{2}{*}{ Gruppe } & \multicolumn{17}{|c|}{ Zahl der Fälle klassiert in die Gruppe: } \\
\hline & & A & $\mathrm{U}$ & $\mathrm{X}$ & $\mathrm{Z}$ & B & V & W & $\mathrm{Y}$ & $\mathrm{C}$ & $\mathrm{P}$ & $\mathrm{Q}$ & $\mathrm{R}$ & $\mathrm{S}$ & $\mathrm{N}$ & 0 & $\mathrm{D}$ & $\mathrm{T}$ \\
\hline & A & 29 & 37 & 27 & 18 & 0 & 0 & 0 & 0 & 0 & 0 & 0 & 0 & 0 & 0 & 0 & 0 & 0 \\
\hline & $\mathrm{U}$ & 6 & 39 & 29 & 10 & 0 & 0 & 0 & 0 & 0 & 0 & 0 & 0 & 0 & 0 & 0 & 0 & 0 \\
\hline & $\mathrm{X}$ & 0 & 8 & 44 & 8 & 0 & 0 & 0 & 0 & 0 & 0 & 0 & 0 & 0 & 0 & 0 & 0 & 0 \\
\hline & Z & 2 & 0 & 8 & 62 & 0 & 0 & 0 & 0 & 0 & 0 & 0 & 0 & 0 & 0 & 0 & 0 & 0 \\
\hline SSA & B & 0 & 0 & 0 & 0 & 43 & 22 & 24 & 0 & 0 & 0 & 0 & 0 & 0 & 1 & 0 & 0 & 0 \\
\hline & V & 0 & 0 & 0 & 0 & 10 & 51 & 20 & 1 & 0 & 0 & 0 & 0 & 0 & 0 & 0 & 0 & 0 \\
\hline & W & 0 & 0 & 0 & 0 & 18 & 16 & 30 & 1 & 0 & 0 & 0 & 0 & 0 & 1 & 0 & 9 & 0 \\
\hline & Y & 0 & 0 & 0 & 0 & 0 & 6 & 0 & 67 & 0 & 0 & 0 & 0 & 0 & 0 & 2 & 0 & 0 \\
\hline HGO & $\mathrm{C}$ & 0 & 0 & 0 & 0 & 0 & 0 & 0 & 0 & 69 & 20 & 7 & 0 & 0 & 0 & 0 & 0 & 0 \\
\hline & P & 0 & 0 & 0 & 0 & 0 & 0 & 0 & 0 & 27 & 57 & 0 & 0 & 0 & 0 & 0 & 0 & 0 \\
\hline & Q & 0 & 0 & 0 & 0 & 0 & 0 & 0 & 0 & 0 & 0 & 69 & 1 & 2 & 0 & 0 & 0 & 0 \\
\hline & $\mathbf{R}$ & 0 & 0 & 0 & 0 & 0 & 0 & 0 & 0 & 0 & 0 & 2 & 97 & 0 & 0 & 0 & 0 & 0 \\
\hline & $S$ & 0 & 0 & 0 & 0 & 0 & 0 & 0 & 0 & 0 & 0 & 0 & 0 & 66 & 0 & 0 & 2 & 0 \\
\hline & $\mathrm{N}$ & 0 & 0 & 0 & 0 & 0 & 0 & 0 & 0 & 0 & 0 & 0 & 0 & 0 & 36 & 0 & 0 & 39 \\
\hline & 0 & 0 & 0 & 0 & 0 & 0 & 0 & 0 & 3 & 0 & 0 & 0 & 0 & 0 & 0 & 69 & 0 & 0 \\
\hline HGA & D & 0 & 0 & 0 & 0 & 0 & 0 & 0 & 0 & 0 & 0 & 0 & 0 & 3 & 32 & 0 & 63 & 30 \\
\hline & $\mathrm{T}$ & 0 & 0 & 0 & 0 & 0 & 0 & 2 & 0 & 0 & 0 & 0 & 0 & 0 & 27 & 0 & 32 & 38 \\
\hline
\end{tabular}


punkte (Tab.4) einer Szene in den verschiedenen Stichproben-Kategorien, und damit kann schließlich deren Bedeckung auf der Erdoberfläche ermittelt werden. Ein Bildpunkt umfaßt in der Orthogonalprojektion auf der Erdoberfläche ein Gebiet von $4514 \mathrm{~m}^{2}$. Sofern eine möglichst genaue Angabe über die Flächenbedeckung durch Schnee gewünscht wird, sollte vor allem in Gebirgsregionen nicht einfach die aus dem Satellitenbild durch Parallelprojektion auf eine Ebene bezogene Fläche gemessen werden, sondern die wahre Oberfläche. Für das Testgebiet (Bergell) beträgt der Unterschied zwischen der wahren Oberfläche und der Orthogonalprojektion ca. $20 \%$. Im Hinblick auf ein operationelles Schneeinformationssystem wäre eine Lösung dahingehend denkbar, daß man die vom Satelliten übermittelte Bildinformation direkt in ein digitales
Reliefmodell projizieren würde. Mit diesem System wäre jederzeit eine exakte Flächenbestimmung von Schnee im Gebirge möglich.

Neben der eigentlichen Klassifikation beinhaltet das erarbeitete System noch zahlreiche vorverarbeitende Programmteile zur geometrischen und radiometrischen Korrektur der Daten sowie zur Ausgabe der Ergebnisse in Form photographischer Bilder. Ein erst kürzlich von der ETHZ erworbenes Bildaufbelichtungsgerät (Optronics Photomation 1700) erlaubt es, die Klassifikationsergebnisse wieder bildmäßig darzustellen und zwar in Bildern von photographischer Qualität.

Bei dem von uns entwickelten Verfahren handelt es sich um eine weitgehend automatisierbare Methode, da lediglich zur Abgrenzung und Auswahl der geeigneten Stichproben (evtl. mit Feldarbeit) ein Interpret

Tabelle 3: Klassifikationsmatrix für die neue Lerngruppenauswahl

\begin{tabular}{|c|c|c|c|c|c|c|c|c|c|c|}
\hline \multirow{7}{*}{ SSO } & \multirow[b]{2}{*}{ Gruppe } & \multicolumn{9}{|c|}{ Zugeordnete Anzahl Bildpunkte zur Lerngruppe: } \\
\hline & & $\mathrm{U}$ & $\mathrm{E}^{\prime}$ & B & $\mathrm{Y}$ & $\mathrm{O}$ & $\mathrm{P}$ & Q & $\mathrm{S}$ & $\mathrm{T}$ \\
\hline & $\mathrm{U}$ & 78 & 6 & 0 & 0 & 0 & 0 & 0 & 0 & 0 \\
\hline & A & 80 & 31 & 0 & 0 & 0 & 0 & 0 & 0 & 0 \\
\hline & $\mathrm{X}$ & 60 & 0 & 0 & 0 & 0 & 0 & 0 & 0 & 0 \\
\hline & Z & 62 & 10 & 0 & 0 & 0 & 0 & 0 & 0 & 0 \\
\hline & $\mathrm{E}$ & 12 & 54 & 0 & 0 & 0 & $4 *$ & 0 & 0 & 0 \\
\hline \multirow[t]{4}{*}{ SSA } & B & 0 & 0 & 87 & 0 & 0 & 0 & 0 & 0 & $3 *$ \\
\hline & V & 0 & 0 & 77 & 4 & 0 & 0 & 0 & 0 & $1^{*}$ \\
\hline & W & 0 & 0 & 58 & 1 & 0 & 0 & 0 & 0 & $16^{*}$ \\
\hline & $\mathrm{Y}$ & 0 & 0 & 4 & 70 & $1^{*}$ & 0 & 0 & 0 & 0 \\
\hline \multirow{7}{*}{ HGO } & $\mathrm{N}$ & 0 & 0 & $9 *$ & 0 & 0 & 0 & 0 & 0 & 66 \\
\hline & $\mathrm{O}$ & 0 & 0 & 0 & $1^{*}$ & 71 & 0 & 0 & 0 & 0 \\
\hline & $\mathrm{P}$ & 0 & 0 & 0 & $1^{*}$ & 0 & 79 & 4 & 0 & 0 \\
\hline & Q & 0 & 0 & 0 & 0 & 0 & 0 & 68 & 4 & 0 \\
\hline & $\mathrm{R}$ & 0 & 0 & 0 & 0 & 0 & 0 & 99 & 0 & 0 \\
\hline & $\mathrm{S}$ & 0 & 0 & 0 & 0 & 0 & 0 & 0 & 67 & 1 \\
\hline & $\mathrm{C}$ & 0 & 0 & 0 & 0 & 0 & 53 & 43 & 0 & 0 \\
\hline \multirow[t]{3}{*}{ HGA } & $\mathrm{T}$ & 0 & 0 & $10 *$ & 0 & 0 & 0 & 0 & 0 & 89 \\
\hline & D & 0 & 0 & $5 *$ & 0 & 0 & 0 & 0 & 3 & 120 \\
\hline & & \multicolumn{4}{|c|}{ Schnee } & \multicolumn{5}{|c|}{ Bildhintergrund } \\
\hline
\end{tabular}

* = Fehlklassierungen von Schnee zum Bildhintergrund oder umgekehrt 
Tabelle 4: Klassifikationsmatrix für den Bildausschnitt

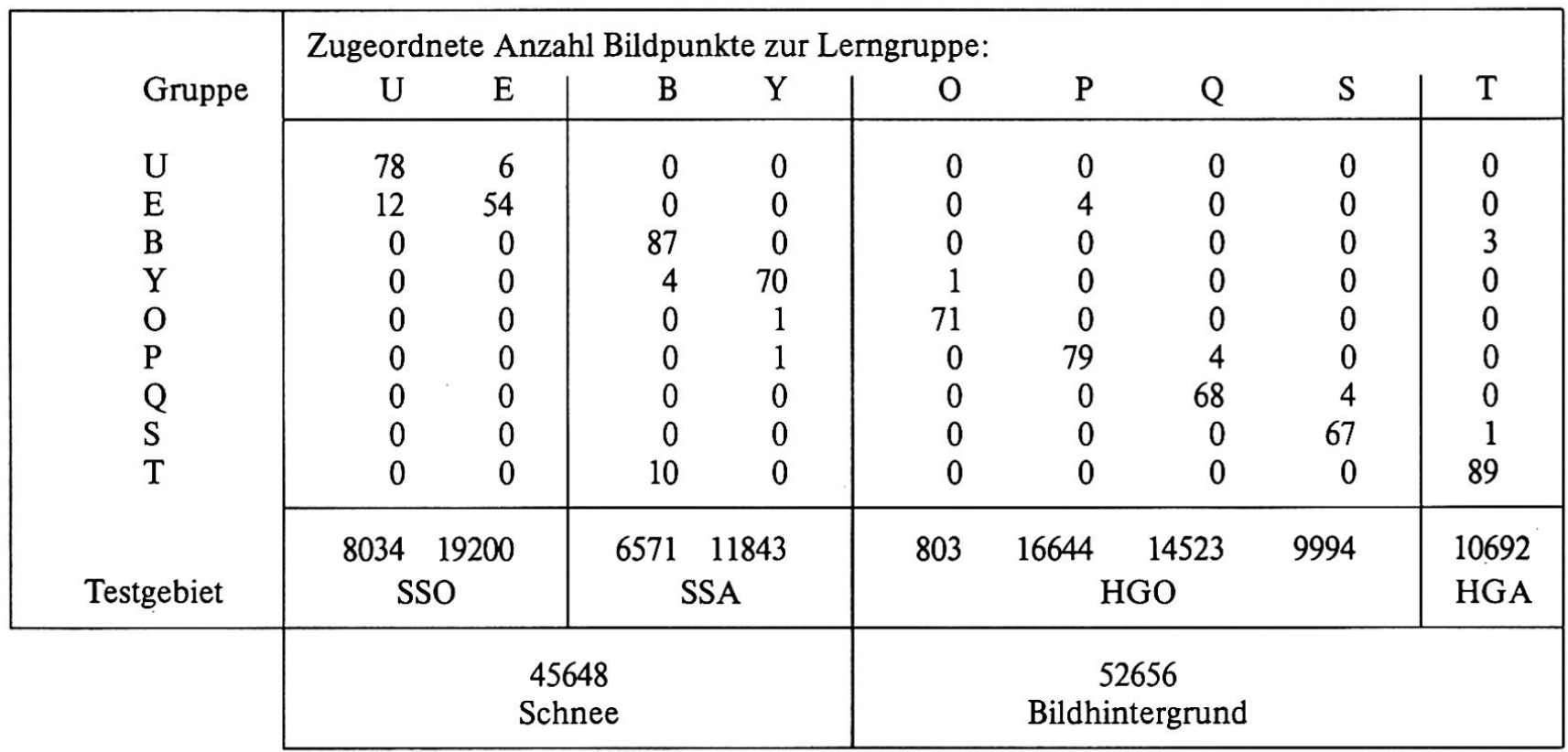

notwendig ist. Es ist aber denkbar, daß nach weiteren Untersuchungen eine umfassend gültige Stichprobensammlung erarbeitet werden kann, so daß auch dieser Schritt automatisiert werden könnte.

Bevor aber ein routinemäßiger Einsatz von automatisierten Systemen in Betracht gezogen wird, ist noch zusätzliche Grundlagenforschung notwendig. Insbesondere setzt die Interpretation von Fernerkundungsdaten hinreichende Kenntnisse über die physikalischen Eigenschaften der Geländeobjekte voraus. Für die Auswertung multispektraler Daten liegen bisher nicht genügend Informationen über das Reflexionsverhalten von Oberflächen vor. Hinzu kommt, daß sowohl die räumliche Struktur von Geländeobjekten als auch die Mischung verschiedener Reflexionssignale innerhalb eines einzelnen Bildelementes die Auswertung erschweren. Neben umfassenden Kenntnissen über die Spektraleigenschaften der Objekte sind wirksamere Methoden zur Elimination von Störeffekten notwendig, wie z. B. von der Atmosphäre verursacht werden.

\section{Literatur}

GFELLER, R., 1975: Untersuchungen zur automatisierten Schneeflächenbestimmung mit Multispektral-Aufnahmen des Erderkundungssatelliten ERTS-1, Diss. Univ. Zürich.

SEIDEL, K., 1975: Analoge und digitale Auswertung von Satellitenbildern (ERTS-1), (Arbeitstitel), in Vorbereitung. 